\title{
A new procedure for power efficiency optimization in microwave ovens based on thermographic measurements and load location search ${ }^{25}$
}

\section{Abstract}

In this work, a novel procedure for increasing the power efficiency of microwave ovens is presented. The procedure consists of iteratively moving the sample within the microwave oven until a maximum average temperature increment is detected across the sample. A new experimental set-up consisting of a multimode microwave cavity, a dielectric tracking system, a microwave power source and a thermographic camera has been built and tested. Results show that it is possible to find optimal positions for the sample within the oven for which microwave power transfer to the sample is maximized.

(c) 2007 Published by Elsevier Ltd.

\section{Introduction}

High power efficiency is one of the most desired characteristics for both industrial and domestic microwave ovens since electric energy is expensive and because low efficiencies indicate that high power reflections may damage the magnetron [1]. In fact, there are many traditional external devices such as tuners, mobile short-circuits, irises, and stubs that may provide adaptation between the microwave source and the final microwave receptor, i.e. sample, achieving low reflections back to the magnetron and maximum power transfer to the sample [2]. Other authors have shown that it is possible to achieve high power efficiencies by using dielectrics around the sample $[3,4]$ or by placing the material to be heated at an optimal position for which the reflection coefficient at the feeding port is minimized [5]. However, all of these devices and methods used for increasing power efficiency are based on the minimization of the so-called reflection coefficient at the feeding port mainly at $2.45 \mathrm{GHz}$ which is the central frequency of the corresponding 2.4$2.5 \mathrm{GHz}$ ISM band.

\footnotetext{
放 Communicated by W.J. Minkowycz.

* Corresponding author.

E-mail address: Juan.Pmolina@upct.es (J.L. Pedreño-Molina).
} 


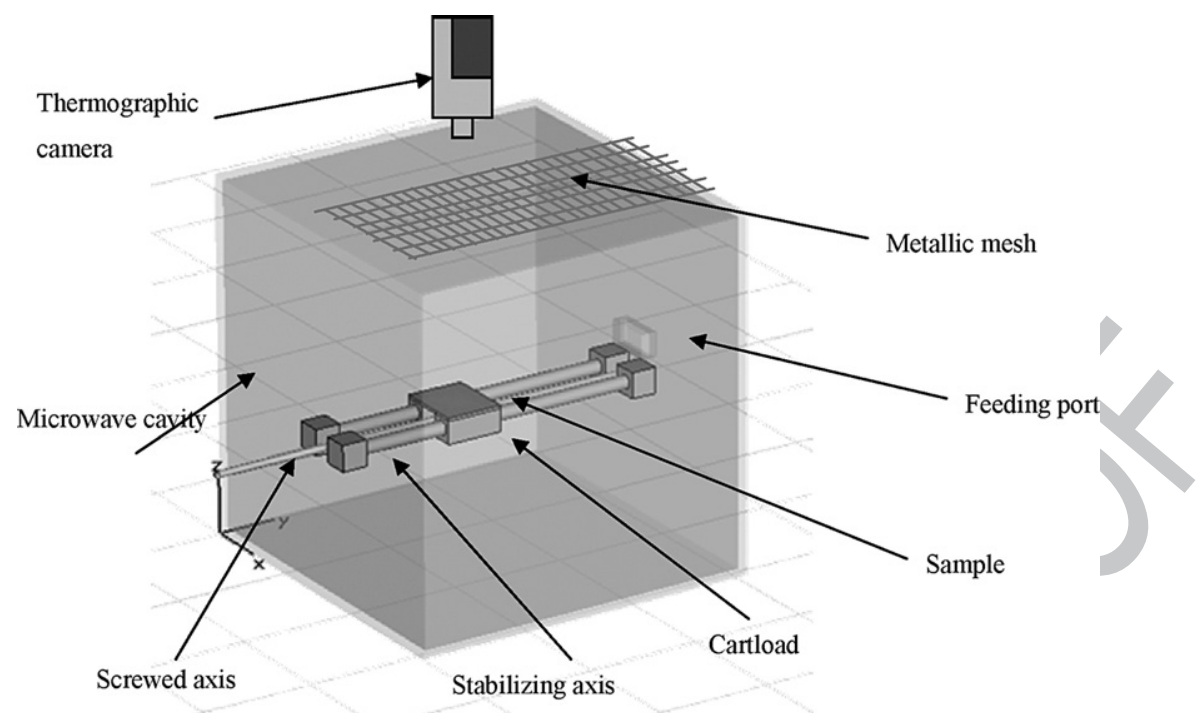

Fig. 1. Experimental set-up used in all tests.

However, it has been recently shown in several works [6,7] that magnetron spectrum is not always centered at that assumed frequency and that, in fact, the spectrum of the magnetron varies with internal temperature, aging, manufacturer model, power reflections and output power. As a result, it seems very difficult to know a priori the output frequency of any magnetron which makes the adapting devices and procedures described in [1-5] difficult to be implemented with guaranties for all magnetron producers or models.

In this work we describe a new method for power efficiency optimization based on an iterative search of sample position within the oven that provides a maximum temperature increase across the sample. This efficiency measurement does not depend on magnetron frequency but on microwave power dissipation and, therefore, it takes into account all the frequency deviation parameters described previously.

\section{Experimental set-up}

Fig. 1 shows a diagram of the experimental set-up used in all tests. The microwave oven consisted of a $60 \times 60 \times 60 \mathrm{~cm}^{3}$ metallic cavity with a metallic mesh placed at its upper wall. This mesh allowed the light crossing the holes but avoided microwaves going out from the oven since all the holes were small enough to act as cut-off waveguides [1].

A

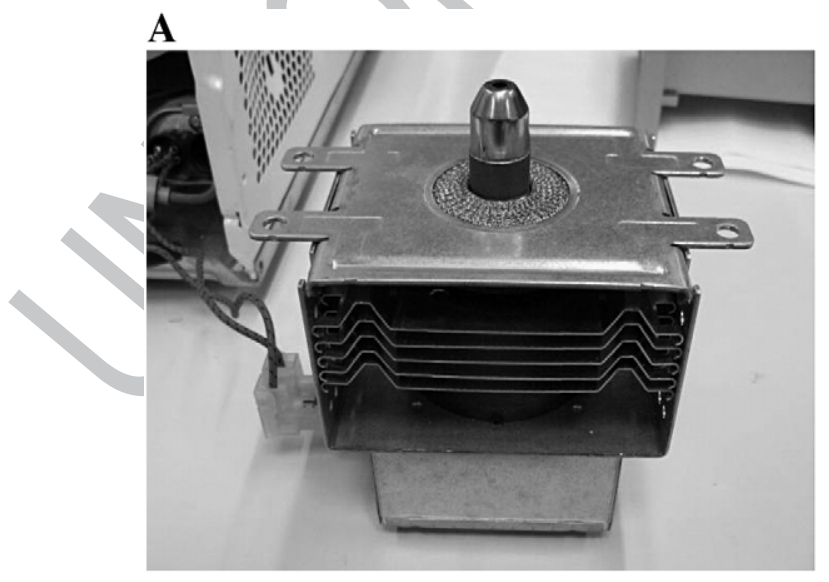

B

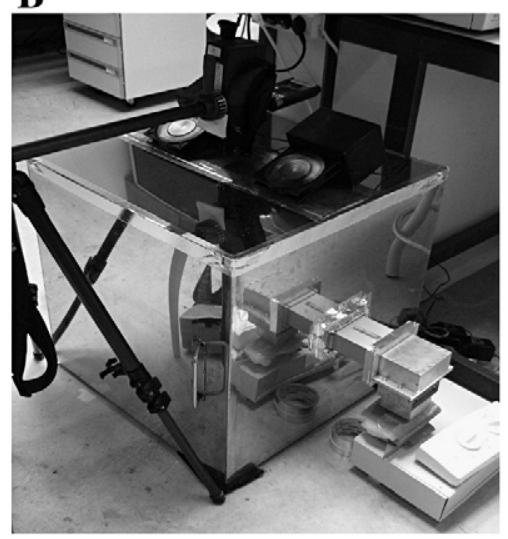

Fig. 2. Details of (A) the employed magnetron and (B) the experimental set-up. 

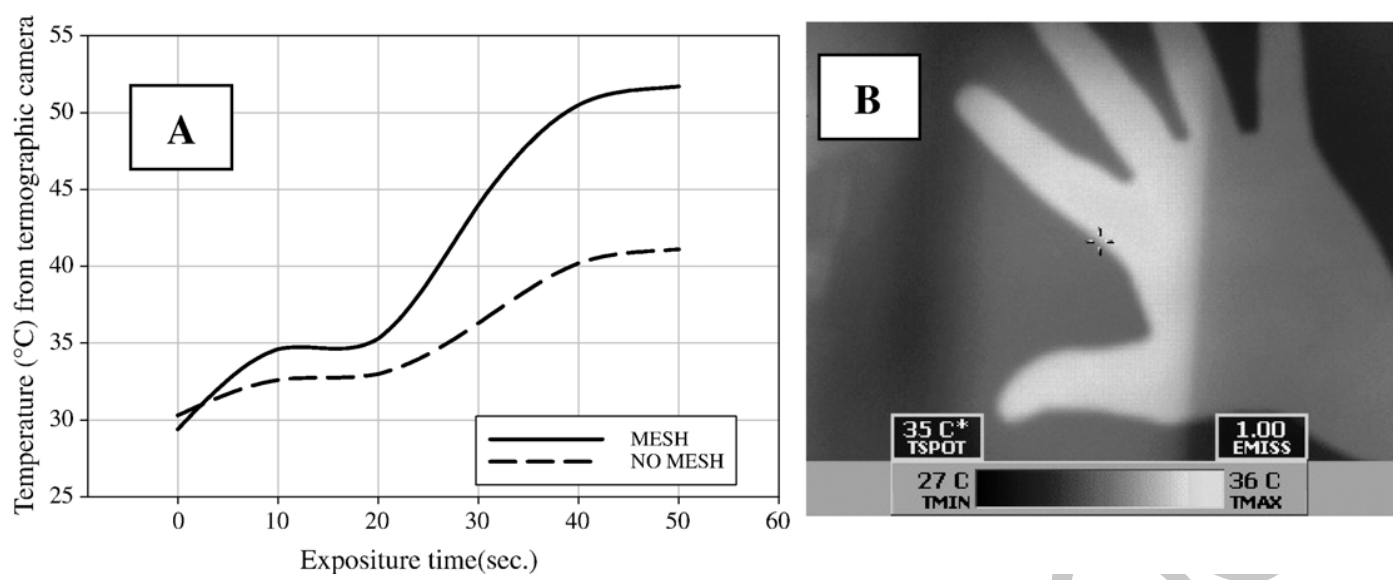

Fig. 3. Influence of the metallic grid on the measured temperature. (A) Average temperature comparison with and without the mesh and (B) effect on the temperature of a hand.

In Fig. 1 one can also observe the tracking system which comprised several nylon pieces such as a cartload, a screwed cylindrical axis that was responsible for the sample movement and two stabilizing pieces for avoiding lateral movements. The dielectric samples were centered at the upper surface of the cartload in all tests. In order to generate microwave energy within the oven a OM75S Samsung magnetron was used and connected to the feeding port, as represented in Fig. 2. The magnetron spectrum showed a central frequency at $2.475 \mathrm{GHz}$. The microwave power level was set to $600 \mathrm{~W}$ and the irradiation time was limited up to $60 \mathrm{~s}$ during all tests in order to avoid the nylon burning. With this configuration, the employed nylon, with relative permittivity data of $\varepsilon^{*}=3.02-j 0.036$, reached its critical temperature at approximately $180 \mathrm{~s}$.

The metallic mesh allows thermograph acquisitions but it also changes the measured temperature since it decreases the amount of light received by the sensor. As a consequence, the temperature measured by the thermographic camera when the metallic grid is interposed must be corrected. Fig. 3A shows average temperature measurements carried out on the same polyester resin sample (sample C), irradiated during different time intervals, with and without mesh. Graphically, the effect produced by the presence of the mesh can be observed in Fig. 3B, where the right part of the hand is occluded by the grid. The employed thermographic camera was a Cyclos PPM LAND model. From Fig. 3, it can be concluded that the introduction of the mesh produces lower temperature measurements than those recorded without the mesh, being that dependence itself a function of the temperature of the sample. It was also detected that the laboratory light was reflected on the metallic walls of the oven, introducing errors during the thermographic measurements. Therefore, several panels were placed on the microwave oven in order to avoid external illumination.

In order to move the cartload, a 1444328 Swiss Mode T11 Maxon DC motor was used. This model, jointly with the screwed axis, provided a linear speed of $11.7 \mathrm{~mm} / \mathrm{s}$ and a precision of $10^{-5} \mathrm{~mm}$ for the sample position. Several software routines were implemented in $\mathrm{C}++$ and Matlab in order to control this motor, allowing precise sample location. The motor system and the controlling PC were connected using a USB interface. During all the tests, a $4 \mathrm{~mm}$ step was employed to locate the sample between $200 \mathrm{~mm}$ and $356 \mathrm{~mm}$ from the magnetron, which provided a total of 40 different positions for each experiment. The minimum and maximum distances from the magnetron were chosen taking into account that results from previous experiments indicated that the optimal positions for the sample would lie inside this interval. Table 1 shows the sample dimensions and permittivity for all the tests. Two geometries were

t1.2 Sample dielectric characteristics and dimensions

\begin{tabular}{lllll}
\cline { 2 - 4 } $\mathrm{t} 1.3$ & & Sample & Dimensions $(\mathrm{cm})$ & Permittivity \\
\cline { 2 - 4 } $\mathrm{t} 1.4$ & $\mathrm{~A}$ & Wood & $4.0 \times 5.2 \times 1.0$ & $1.99-j 0.19$ \\
$\mathrm{t} 1.5$ & $\mathrm{~B}$ & Epoxy Resin & $4.3 \times 8.6 \times 1.0$ & $4.5488-j 1.4986$ \\
$\mathrm{t} 1.6$ & $\mathrm{C}$ & Polyester resin & $4.3 \times 8.6 \times 1.0$ & $4.3825-j 0.4984$ \\
$\mathrm{t} 1.7$ & $\mathrm{D}$ & Polyester resin (cylindrical) & Radius $=5.5$, thickness $=2.5$ \\
\hline
\end{tabular}




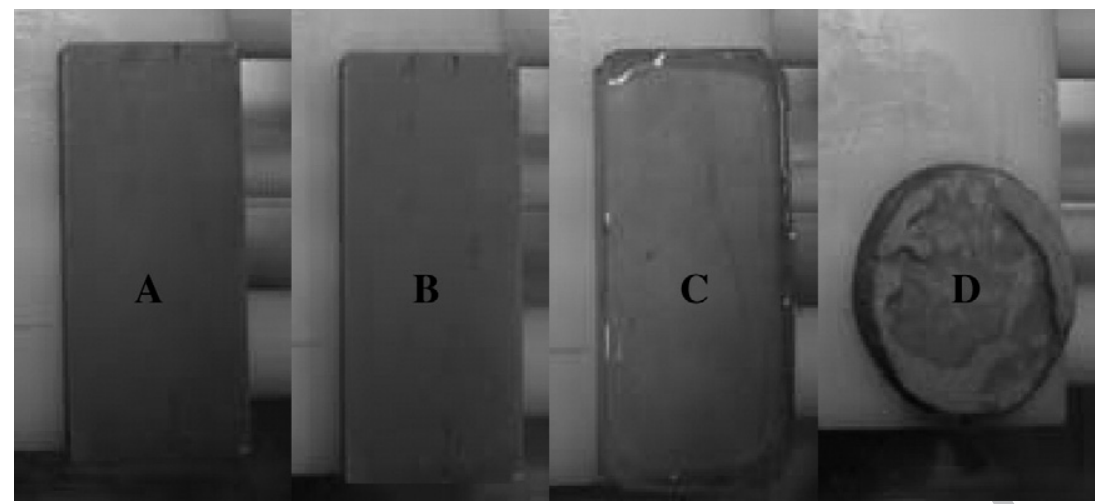

Fig. 4. Employed samples with different materials: (A) wood, (B) and (D) polyester resin and (C) epoxy resin.

used: rectangular and cylindrical and several materials, such as epoxy, polyester resin and wood were utilized, as Fig. 4 shows.

\section{Description of the power efficiency optimization procedure}

The developed optimization procedure is based on the fact that when the microwave power absorbed by the sample is maximum, the average temperature increment at the sample is maximum too [1]. If the incident power level and
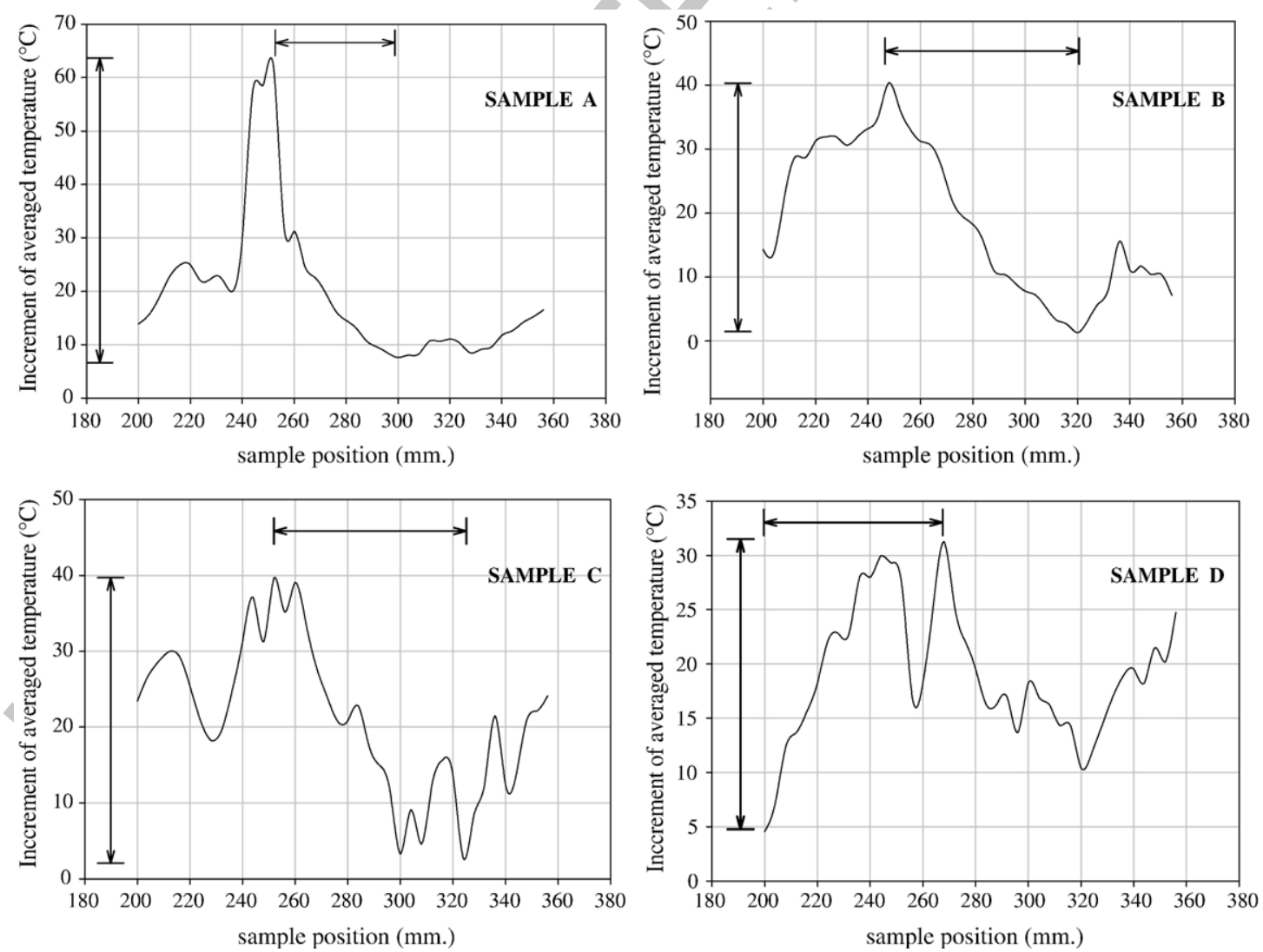

Fig. 5. Evolution of the temperature increment during optimization procedure in samples A, B, C and D. 
t2.2 Summary of the obtained results with the optimization procedure

\begin{tabular}{llllll}
$\mathrm{t} 2.3$ & Sample & $\Delta T_{\mathrm{a}}\left({ }^{\circ} \mathrm{C}\right)$ & $\Delta$ Pos $(\mathrm{mm})$ & Exposure time $(\mathrm{s})$ & Optimal position $(\mathrm{mm})$ \\
\cline { 2 - 6 } $\mathrm{t} 2.4$ & $\mathrm{~A}$ & 54.7 & 48 & 20 & 252 \\
$\mathrm{t} 2.5$ & $\mathrm{~B}$ & 39.1 & 72 & 60 & 248 \\
$\mathrm{t} 2.6$ & $\mathrm{C}$ & 37.1 & 72 & 60 & 252 \\
$\mathrm{t} 2.7$ & $\mathrm{D}$ & 26.7 & 68 & 60 & 268 \\
\hline
\end{tabular}

irradiation time are kept constant, the sample position that provides a higher temperature increment is the location that provides the best microwave cavity efficiency.

The procedure comprises the following steps: (a) motor movement in order to place the sample at a new position, (b) acquisition of initial sample temperature, (c) microwave irradiation at a constant power level and during constant time intervals, (d) measurement of sample final temperature distribution, (e) cooling the sample with fans in order to avoid sample overheating, (f) back to step (a) until last position is reached, (g) determination of the optimal position for maximum heating efficiency by detecting the highest peak in the curve representing temperature increment against the sample position.

\section{Results and discussion}

In order to test the procedure described above, the autonomous process has been carried out for each of the four samples. The epoxy and polyester samples have been irradiated, at each of the 40 different positions, with a power of $600 \mathrm{~W}$ during $60 \mathrm{~s}$. At every position, thermographic images have been acquired and from them, the average temperatures have been obtained and converted to real temperatures by means of the calibration curve from Fig. 3A. Thus, in Fig. 5 the evolution of the average temperature for each sample as a function of sample position is shown. In this figure, the effect of the metallic mesh has been compensated. The $y$ axis represents the increment of the average temperature with respect to the initial sample temperature after microwave irradiation. On the other hand, $x$ axis indicates the position at which the sample was irradiated.

Since a long heating time could produce a destructive effect on the wood sample, a short irradiation time ( $20 \mathrm{~s})$ has been considered during this test. In the figure, it can be observed a very high increment of the average temperature when the sample is at the optimal position. It is noticeable that a displacement of only $5 \mathrm{~cm}$ from the optimal position can dramatically decrease the heating efficiency. Similar curves have been obtained for the rest of the samples - B, C and D - in order to verify the methodology when different materials, sizes and shapes are used. There, irradiation times of $60 \mathrm{~s}$ have been applied for the heating process. One can also deduce from Fig. 5 that there are sample positions at which microwave absorption and power efficiency are minimum since average temperature increments are negligible. This indicates that sample location is of the utmost importance when trying to design an efficient microwave oven.

In order to summarize the obtained results, Table 2 shows the average temperature difference $\left(\Delta T_{\mathrm{a}}\right)$ between the optimal position and the position at which the reached average temperature is lower. $\Delta$ Pos indicates the distance in millimeters between these two positions. The optimal position (distance from the magnetron) for maximum efficiency is also indicated in millimeters. From these data one can conclude that microwave oven efficiency is very sensitive to sample location and that very short sample movements can lead to high energy losses or savings.

Finally, from the analysis of the influence of the size and material employed in the microwave heating process, it can be concluded that different sample dielectric permittivity and sizes produce different levels of efficiency, although an optimal position can be similarly found for all the tested materials.

These results agree well with the findings reported in [5] although in that work efficiency was measured at low microwave power levels, with a slightly different experimental set-up and at different frequency values. In fact, in [5] it was reported that the reflection coefficient, which compares the reflected microwave wave versus the incident one, was minimum at different sample locations depending on several factors such as permittivity, frequency and geometry. The same behaviors are also observed in this work.

\section{Conclusions}

In this paper, a novel methodology for finding the optimal placement of the sample for maximum heating efficiency inside a microwave heating cavity is described. This methodology is based on a process that iteratively places the sample at different distances from the magnetron, then irradiates it at high power levels and determines the temperature increment from thermographic images. The use of infrared cameras for temperature measurements allows avoiding the 
problems associated with frequency shifts of the magnetron since, in this case, efficiency is measured through microwave power dissipation, which is very closely related to temperature increment.

For the experimental testing of the proposed methodology, a microwave cavity with automated sample positioning has been constructed, and several experiments have been carried out using samples of different sizes, materials and shapes. The obtained results confirm not only that an optimal position can be found at which heating efficiency is maximum, but also that a displacement of a few centimeters from this optimum position can considerably reduce the heating efficiency.

\section{References}

[1] A.C. Metaxas, R.J. Meredith, Industrial Microwave Heating, Peter Peregrinus, London, 1983, pp. $109-112$.

[2] R.J. Meredith, Engineers' Handbook of Industrial Microwave Heating, Power and Energy Series, IEE, Stevenage, 1998 , pp. 83-149.

[3] J. Monzó-Cabrera, A. Díaz-Morcillo, J.L. Pedreño-Molina, D. Sánchez-Hernández, Microwave and Optical Technology Letters 40 (4) (2004) $319-322$.

[4] J. Monzó-Cabrera, J. Escalante, A. Díaz-Morcillo, A. Martínez-González, D. Sánchez-Hernández, Microwave and Optical Technology Letters 41 (5) (2004) 414-417.

[5] M.E. Requena-Pérez, J.L. Pedreño-Molina, J. Monzó-Cabrera, A. Díaz-Morcillo, IEEE Transactions on Microwave Theory and Techniques 53 (6) (2005) 2114-2120.

[6] V.B. Neculaes, R.M. Gilgenbach, Y.Y. Lau, Applied Physics Letters 83 (2003) 1938-1940.

[7] V.B. Neculaes, R.M. Gilgenbach, Y.Y. Lau, M.C. Jones, W.M. White, IEEE Transactions On Plasma Science 32 (3) (2004) $1152-1159$. 